\title{
Synthesis of Functionalized Dihydropyrido[2,3-d]pyrimidines in Aqueous Medium
}

\author{
Saeed Balalaie*a,b \\ Hamed Esmaeilabadia \\ Saber Mehrparvar ${ }^{a}$ \\ Frank Rominger ${ }^{c}$ \\ Fatima Hamdana \\ Hamid Reza Bijanzadeh ${ }^{d}$ \\ a Peptide Chemistry Research Center, K. N. Toosi University of \\ Technology, P. O. Box 15875-4416, Tehran, Iran \\ balalaie@kntu.ac.ir \\ ${ }^{b}$ Medical Biology Research Center, Kermanshah University of \\ Medical Sciences, Kermanshah, Iran \\ c Organisch-Chemisches Institut der Universität Heidelberg, \\ Im Neuenheimer Feld 270, 69120 Heidelberg, Germany \\ d Department of Biophysics, Tarbiat Modares University, \\ Tehran, Iran
}

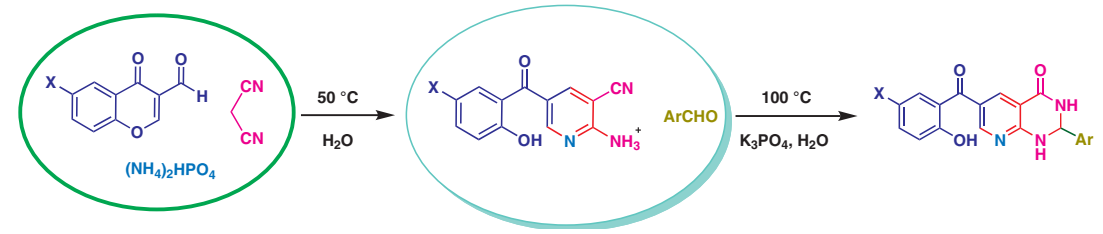

Dedicated to Prof. Dr. Uli Kazmaier on the occasion of his birthday

Received: 08.08.2017

Accepted after revision: 27.11.2017

Published online: 18.01 .2018

DOI: 10.1055/s-0036-1591869; Art ID: so-2017-d0049-I

License terms: cc)

Abstract Synthesis of functionalized 2,3-dihydropyrido[2,3-d]pyrimidin-4(1H)-one from a cascade reaction between 3-formylchromone, malononitrile, diammonium hydrogen phosphate, and aromatic aldehydes in aqueous media is described.

Keywords 2-aminopyridine, dihydropyrido[2,3-d]pyrimidine, aqueous medium

One of the fundamental challenges and ultimate goals for organic chemists is to perform reactions under green reaction conditions, such as carrying out the reactions in water to reduce use of organic solvents and to develop environmentally friendly processes. ${ }^{1,2}$ Designing a novel posttransformational reaction in aqueous medium to access functionalized compounds has received much interest in the synthesis of organic compounds., 3

2-Aminopyridines are known skeletons in organic and medicinal chemistry. ${ }^{5}$ Methods for the synthesis of 2-aminopyridines involve the reaction of 2-halopyridines with amines using metal catalysts. ${ }^{6-9}$ Since in some cases, amination leads to a mixture of products and the reported methods are often not applicable at large scale, developing new and mild methods for the preparation of 2-aminopyridines is still desirable. Certain 2-amino-3-cyano-pyridines show bioactivity, and different approaches for their synthesis have been reported. ${ }^{10}$ In particular, 3-formyl chromone has been used as a starting material for the synthesis 2amino-3-cyano-pyridines through ring opening. ${ }^{11}$ Such compounds have a potential for cyclization to access heterocyclic skeletons such as quinazolinones. ${ }^{12,13}$ Recently, Langer reported the synthesis of 2,3-dihydroquinazolinones using 2-aminobenzonitriles in the presence of a base in aqueous medium. ${ }^{14}$ Quinazolinones have extensive biological properties such as anticancer, antibacterial, antihypertensive, antidiabetic, anti-inflammatory, anticonvulsant, and antiallergic activities. ${ }^{15,16}$ In Figure 1, the structures of some bioactive compounds containing the quinazolinone skeleton are shown.

In view of the importance of functionalized quinazolinones, ${ }^{17}$ we report herein the synthesis of 2,3-dihydropyrido[2,3-d]pyrimidin-4(1H)-one through a post-transformation of a multicomponent reaction in water with diammonium hydrogen phosphate (DAHP) ${ }^{18}$ as a source of nitrogen (Scheme 1). The three-component reaction of chromone carbaldehyde, malononitrile, and diammonium hydrogen phosphate (DHAP) in water led to functionalized 2-amino-

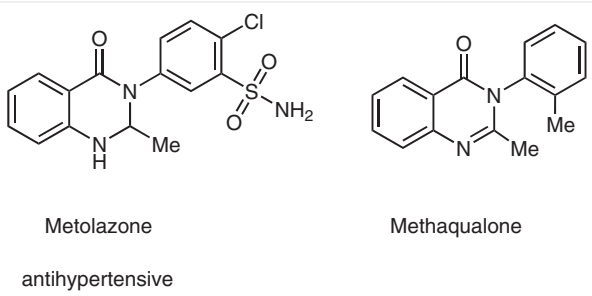

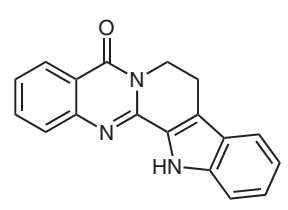

Rutaecarpine

sedative, withdraw<smiles>COc1cccc(C2NC(=O)c3cc(Cl)ccc3N2)c1</smiles>

anticancer activity

Figure 1 The structure of some bioactive compounds with quinazolinone skeleton 


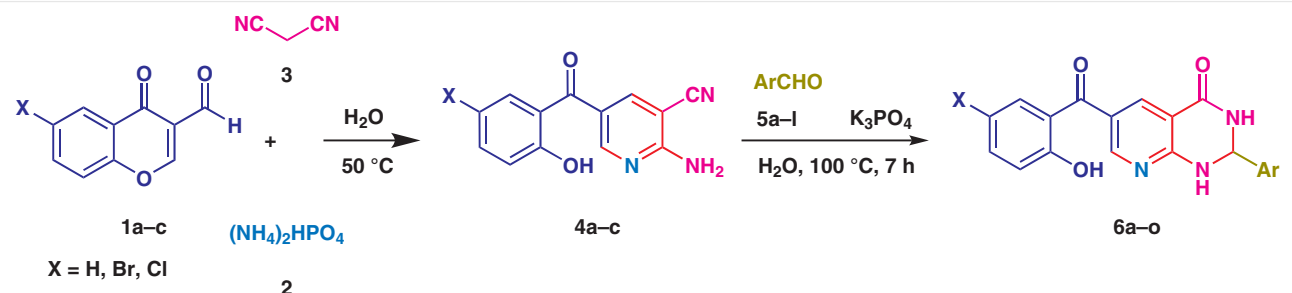

Scheme 1 Synthesis of 2,3-dihydropyrido[2,3-d]pyrimidin-4(1H)-ones 6a-o

pyridines $\mathbf{4 a - c}$ and their subsequent reaction with aromatic aldehydes in the presence of potassium phosphate in water led to the desired 2,3-dihydropyrido[2,3-d]pyrimidin4(1H)-ones 6a-o.

At first, 3-formylchromones 1a-c were synthesized based on a known reaction of 2-hydroxyacetophenes and Meldrum's acid, and the study began with designing a model three-component reaction of 3-formylchromone $\mathbf{1 a}$, malononitrile $\mathbf{2}$, and a source of nitrogen $\mathbf{3}$ in water at room temperature or $50{ }^{\circ} \mathrm{C}$. Under all reaction conditions, the isolated product was compound $\mathbf{4 a}$. As shown in Table 1, diammonium hydrogen phosphate (DAHP), ammonium acetate, and ammonia solution were used as the sources of nitrogen. Comparison of the yield of the product and reaction temperature showed that DAHP was the most suitable nitrogen source and the optimal temperature was $50{ }^{\circ} \mathrm{C}$. Meanwhile, the molar ratio of DAHP was investigated and the best yield was obtained with 1.5 equiv of DAHP (93\% yield). The reaction was also carried out in ethanol, but using water resulted in a better yield. The driving force of the reaction is the precipitation of the product from water. After optimizing the reaction conditions, the scope and limitations were studied using different 3-formylchromones 1a-c, malononitrile, and diammonium hydrogen phosphate. The desired products $\mathbf{4 a - c}$ were synthesized under the optimized conditions and the results are summarized in Scheme 2 and Table $1 .{ }^{19}$

The structures of compounds $\mathbf{4 a - c}$ were deduced from their ${ }^{1} \mathrm{H}$ and ${ }^{13} \mathrm{C}$ NMR spectroscopic and ESI-HRMS data. For compound $\mathbf{4 a}$ as a representative example, the ${ }^{1} \mathrm{H}$ NMR spectrum consisted of singlets for the $-\mathrm{OH}$ and $-\mathrm{NH}_{2}$ protons at $\delta=10.25$ and $7.81 \mathrm{ppm}$, respectively. The $\mathrm{H}-2$ and $\mathrm{H}-4$ of the pyridine ring resonated at $\delta=8.11$ and $8.47 \mathrm{ppm}$. The proton decoupled ${ }^{13} \mathrm{C}$ NMR spectrum of $4 a$ showed 13 distinct resonances, consistent with the proposed structure. The carbonyl carbon of the unsaturated ketone reso-
Table 1 Optimization of Reaction Conditions for the Synthesis of Functionalized 2-Aminopyridine $\mathbf{4 a}$

\begin{tabular}{lllll}
\hline Entry & Solvent & Nitrogen source (molar ratio) & Temp. $\left({ }^{\circ} \mathrm{C}\right)$ & Yield (\%) \\
\hline 1 & $\mathrm{H}_{2} \mathrm{O}$ & $\left(\mathrm{NH}_{4}\right)_{2} \mathrm{HPO}_{4}(0.5)$ & 50 & 78 \\
2 & $\mathrm{H}_{2} \mathrm{O}$ & $\left(\mathrm{NH}_{4}\right)_{2} \mathrm{HPO}_{4}(1.0)$ & 50 & 90 \\
3 & $\mathrm{H}_{2} \mathrm{O}$ & $\left(\mathrm{NH}_{4}\right)_{2} \mathrm{HPO}_{4}(0.5)$ & $\mathrm{RT}$ & 70 \\
4 & $\mathrm{H}_{2} \mathrm{O}$ & $\left(\mathrm{NH}_{4}\right)_{2} \mathrm{HPO}_{4}(1.0)$ & $\mathrm{RT}$ & 74 \\
5 & $\mathrm{H}_{2} \mathrm{O}$ & $\left(\mathrm{NH}_{4}\right)_{2} \mathrm{HPO}_{4}(1.5)$ & $\mathrm{RT}$ & 79 \\
$\mathbf{6}$ & $\mathrm{H}_{2} \mathrm{O}$ & $\left(\mathbf{N H}_{4}\right)_{2} \mathrm{HPO}_{4} \mathbf{( 1 . 5 )}$ & $\mathbf{5 0}$ & $\mathbf{9 3}$ \\
7 & $\mathrm{H}_{2} \mathrm{O}$ & ammonium acetate (1.0) & 50 & 88 \\
8 & $\mathrm{H}_{2} \mathrm{O}$ & ammonium acetate (1.0) & $\mathrm{RT}$ & 83 \\
9 & $\mathrm{H}_{2} \mathrm{O}$ & ammonia 25\% (0.5) & 50 & 70 \\
10 & EtOH & ammonium acetate (0.5) & 50 & 75 \\
11 & EtOH & ammonia 25\% (1.0) & 50 & 72 \\
\hline
\end{tabular}

nated at $\delta=192.2 \mathrm{ppm}$. The structure of 4a was subsequently confirmed by single-crystal X-ray crystallographic analysis (Figure 2). Compound 4a can form effective intermolecular hydrogen bonding in the crystal structure between the $-\mathrm{NH}_{2}$ and $-\mathrm{CN}$ groups and the pyridine nitrogen (see the Supporting Information)

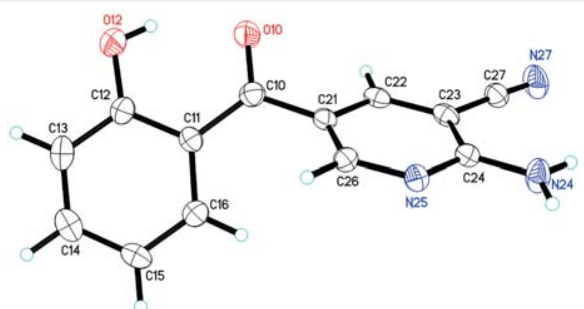

Figure 2 ORTEP structure of $\mathbf{4 a}$

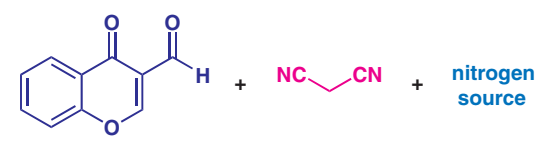

1a

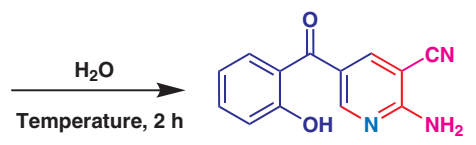

$4 a$

Scheme 2 Synthesis of functionalized 2-aminopyridines $\mathbf{4 a - c}$ through three-component reaction in water 
After the synthesis of compounds $\mathbf{4 a - c}$, the model reaction was carried out using $\mathbf{4 a}$ and benzaldehyde in water and in the presence of different bases, when the desired 2,3-dihydropyrido[2,3-d]pyrimidin-4(1H)-one 6a was isolated. In an effort to optimize the reaction, different bases such as trimethylamine, diisopropylethylamine, L-proline, potassium hydroxide, potassium carbonate, DAHP, cesium carbonate and potassium phosphate were studied, with the best yield being obtained with potassium phosphate. After finding the most suitable base, the model reaction was investigated in a range of solvents such as acetonitrile, ethanol, and DMF as well as water. However, in all cases, the yield of the desired product was lower compared with using water as the solvent. The model reaction was then investigated using varying amounts of potassium phosphate as the base and the optimum yield of $86 \%$ was obtained with 1.5 equivalents. Subsequently, the influence of the reaction temperature was investigated and it was found that $100{ }^{\circ} \mathrm{C}$ resulted in the best yield. Thus, the optimal reaction conditions for the synthesis of $\mathbf{6} \mathbf{a}$ involved conducting the reaction in water with 1.5 equivalents of potassium phosphate at $100{ }^{\circ} \mathrm{C}$ (Table 2 ).

Table 2 Optimization of the Reaction Conditions for the Synthesis of $6 a^{a}$

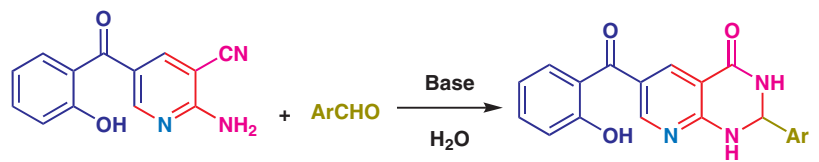

$4 a$

$5 a$

$6 a$

\begin{tabular}{lllll}
\hline Entry & Base & $\begin{array}{l}\text { Concentration } \\
(\text { molar ratio })\end{array}$ & Temp. $\left({ }^{\circ} \mathrm{C}\right)$ & Yield $(\%)$ \\
\hline 1 & $\mathrm{Et}_{3} \mathrm{~N}$ & 1 & 100 & 72 \\
2 & DIPEA & 1 & 100 & 75 \\
3 & L-Proline & 1 & 100 & 52 \\
4 & $\mathrm{NaOH}$ & 1 & 100 & 47 \\
5 & $\left(\mathrm{NH}_{4}\right)_{2} \mathrm{HPO}_{4}$ & 1 & 100 & 50 \\
6 & $\mathrm{Cs}_{2} \mathrm{CO}_{3}$ & 1 & 100 & 62 \\
7 & $\mathrm{~K}_{2} \mathrm{CO}_{3}$ & 1 & 100 & 59 \\
8 & $\mathrm{~K}_{3} \mathrm{PO}_{4}$ & 0.2 & 100 & 43 \\
9 & $\mathrm{~K}_{3} \mathrm{PO}_{4}$ & 0.5 & 100 & 56 \\
10 & $\mathrm{~K}_{3} \mathrm{PO}_{4}$ & $\mathbf{1 . 5}$ & $\mathbf{1 0 0}$ & $\mathbf{8 6}$ \\
11 & $\mathrm{~K}_{3} \mathrm{PO}_{4}$ & 2 & 100 & 86 \\
12 & $\mathrm{~K}_{3} \mathrm{PO}_{4}$ & 1.2 & $\mathrm{RT}$ & 47 \\
13 & $\mathrm{~K}_{3} \mathrm{PO}_{4}$ & 1.2 & 50 & 65 \\
\hline
\end{tabular}

a Reaction conditions: 2-aminopyridine $(1 \mathrm{mmol}, 239 \mathrm{mg})$, benzaldehyde $(1.2 \mathrm{mmol}, 127 \mathrm{mg}), \mathrm{K}_{3} \mathrm{PO}_{4}(1.5 \mathrm{mmol}, 340 \mathrm{mg})$ in $\mathrm{H}_{2} \mathrm{O}(6 \mathrm{~mL})$. In all cases, the reaction time was $7 \mathrm{~h}$.
After finding suitable reaction conditions for the synthesis of $\mathbf{6 a}$, the scope of the reaction was explored using different functionalized 2-aminopyridines and aromatic aldehydes. The product 2,3-dihydropyrido[2,3-d]pyrimidin$4(1 H)$-ones $\mathbf{6 a - 0}$ were obtained in moderate to good yields and with no side reactions (Table 3 ). A characteristic resonance for all of these compounds in the ${ }^{1} \mathrm{H}$ NMR spectra was a singlet at $\delta=5.90-6.10 \mathrm{ppm}$ assigned to the aliphatic methine proton and also a distinctive peak in the ${ }^{13} \mathrm{C}$ NMR spectra for the $\mathrm{sp}^{3}$ carbon at $\delta=64.0-65.0 \mathrm{ppm}$. The ${ }^{13} \mathrm{C}$ NMR spectra of $\mathbf{6 a - 0}$ exhibited characteristic signals in the $\delta=161.0-163.0$ and 192.0-195.0 ppm region associated with the carbonyl carbons of the amide and ketone moieties. There is a suitable disposition for hydrogen bonding between the carbonyl group and the phenolic hydroxyl, with the latter resonating at $\delta=10.20-10.50 \mathrm{ppm}$ in the ${ }^{1} \mathrm{H}$ NMR spectra. ${ }^{20}$

Subsequently, we investigated one-pot reaction conditions for the reaction model without separation of $\mathbf{4 a}$ and the second reaction was carried out by adding potassium phosphate to the aqueous reaction medium. This led to formation of the desired product 6a with a lower yield of $70 \%$ compared with $86 \%$.

In conclusion, we have reported a novel approach to access functionalized 2,3-dihydropyrido[2,3-d]pyrimidin$4(1 H)$-ones $\mathbf{6 a - 0}$ in good yields through sequential reaction of functionalized 2-aminopyridines $\mathbf{4 a - c}$, which were synthesized through a three-component reaction in water in which diammonium hydrogen phosphate was used as a source of nitrogen in the reaction mixture. The current strategy offers advantages such as moderate to good yields, purity of products, simple work-up, and use of an environmentally benign solvent.

\section{Funding Information}

We gratefully acknowledge the Iran National Science Foundation (INSF) for financial support.

\section{Supporting Information}

Supporting information for this article is available online at https://doi.org/10.1055/s-0036-1591869. It contains figures, tables, and CIF files for ${ }^{1} \mathrm{H}$ NMR, ${ }^{13} \mathrm{C}$ NMR, IR, and HRMS spectra for compounds $4 \mathbf{a}$ and $\mathbf{6 a - 0}$ and X-ray crystal data for compounds $\mathbf{4 a}$.

\section{References and Notes}

(1) Cioc, R. C.; Ruijter, E.; Orru, R. V. A. Green Chem. 2014, 16, 2958; and references cited therein

(2) (a) Zhang, W.; Gue, B. W. Jr. Green Techniques for Organic Synthesis and Medicinal Chemistry; Wiley\&Sons: Chiechester, 2012. (b) Science of Synthesis Water in Organic Synthesis; Kobayashi, S., Ed.; Thieme Verlag: Stuttgart, 2012. (c) Simon, M.-O.; Li, C.-J. 
Table 3 Synthesis of 2,3-Dihydropyrido[2,3-d]pyrimidin-4(1H)-one 6a-o in Aqueous Medium ${ }^{\text {a }}$

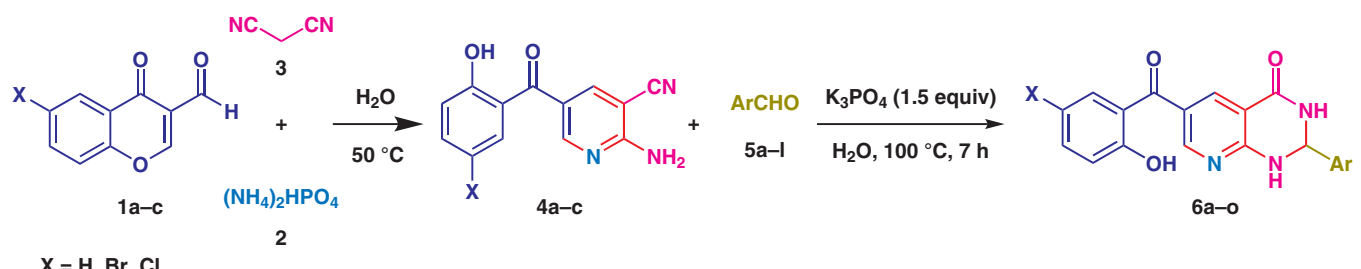

\begin{tabular}{|c|c|c|c|}
\hline Product & $x$ & $\mathrm{Ar}$ & Yield (\%) \\
\hline $6 a$ & $\mathrm{H}$ & $4-\mathrm{ClC}_{6} \mathrm{H}_{4}$ & 82 \\
\hline $6 b$ & $\mathrm{H}$ & 4- $\mathrm{MeOC}_{6} \mathrm{H}_{4}$ & 61 \\
\hline $6 c$ & $\mathrm{H}$ & $4-\mathrm{BrC}_{6} \mathrm{H}_{4}$ & 80 \\
\hline $6 d$ & $\mathrm{H}$ & $4-\mathrm{O}_{2} \mathrm{NC}_{6} \mathrm{H}_{4}$ & 61 \\
\hline $6 e$ & $\mathrm{H}$ & $\mathrm{C}_{6} \mathrm{H}_{5}$ & 79 \\
\hline $6 f$ & $\mathrm{H}$ & 4- $-\mathrm{FC}_{6} \mathrm{H}_{4}$ & 81 \\
\hline $6 g$ & $\mathrm{H}$ & 4- $\mathrm{NCC}_{6} \mathrm{H}_{4}$ & 77 \\
\hline $6 \mathrm{~h}$ & $\mathrm{H}$ & 2- $\mathrm{ClC}_{6} \mathrm{H}_{4}$ & 83 \\
\hline $6 i$ & $\mathrm{Cl}$ & $4-\mathrm{ClC}_{6} \mathrm{H}_{4}$ & 71 \\
\hline $6 \mathbf{j}$ & $\mathrm{Br}$ & $4-\mathrm{ClC}_{6} \mathrm{H}_{4}$ & 67 \\
\hline $6 k$ & $\mathrm{H}$ & $2-\mathrm{BrC}_{6} \mathrm{H}_{4}$ & 73 \\
\hline $6 I$ & $\mathrm{H}$ & 4- $\mathrm{MeC}_{6} \mathrm{H}_{4}$ & 65 \\
\hline $6 m$ & $\mathrm{Cl}$ & 4- $\mathrm{MeOC}_{6} \mathrm{H}_{4}$ & 56 \\
\hline $6 n$ & $\mathrm{H}$ & 3-pyridyl & 71 \\
\hline 60 & $\mathrm{H}$ & 2-thienyl & 89 \\
\hline
\end{tabular}

a In all cases, the reaction time was $7 \mathrm{~h}$.

Chem. Soc. Rev. 2012, 41, 1415. (d) Chanda, A.; Fokin, V. V. Chem. Rev. 2009, 109, 725. (e) Li, C.-J.; Chen, L. Chem. Soc. Rev. 2006, 35, 68.

(3) (a) Green Synthetic Approaches for Biologically Relevant Heterocycles; Brahmachari, G., Ed.; Elsevier: Amsterdam, 2014. (b) Aqueous Microwave Assisted Chemistry, Synthesis and Catalysis; Polshettiwar, V.; Varma, R. S., Eds.; RSC Publishing: Cambridge, 2010.

(4) (a) Green Chemistry: Synthesis of Bioactive Heterocycles; Ameta, K. L.; Dandia, A., Eds.; Springer: India, 2014. (b) Balalaie, S.; Ramezani, Kejani. R.; Ghabraie, E.; Darvish, F.; Rominger, F.; Hamdan, F.; Bijanzadeh, H. R. J. Org. Chem. 2017, 82, 12141. (c) Balalaie, S.; Mirzaie, S.; Nikbakht, A.; Hamdan, F.; Rominger, F.; Navari, R.; Bijanzadeh, H. R. Org. Lett. 2017, 19, 6124.

(5) (a) Hilton, S.; Naud, S.; Caldwell, J.; Boxall, K.; Burns, S.; Anderson, V. E.; Antoni, L.; Allen, C. E.; Pearl, L. H.; Oliver, A. W.; Aherne, G. W.; Garrett, M. D.; Collins, I. Bioorg. Med. Chem. 2010, 459. (b) Yonezawa, S.; Tamura, Y.; Kooriyama, Y.; Sakaguchi, G. PCT Int. Appl. WO2010047372, 2010. (c) Steinig, A. G.; Mulvihill, M. J.; Wang, J.; Werner, D. S.; Weng, Q.; Kan, J.; Coate, H.; Chen, X. U. S. Pat. Appl. US2009197862, 2009.

(6) (a) Goldstein, D. M.; Gong, L.; Michoud, C.; Palmer, W. S.; Sidduri, A. PCT Int. Appl. WO 2008028860, 2008. (b) Kling, A.; Backfisch, G.; Delzer, J.; Geneste, H.; Graef, C.; Hornberger, W.;
Lange, U. E. W.; Lauterbach, A.; Seitz, W.; Subkowski, T. Bioorg. Med. Chem. 2003, 11, 1319. (c) Bolliger, J. L.; Oberholzer, M.; Frech, C. M. Adv. Synth. Catal. 2011, 353, 945.

(7) (a) Yang, B. H.; Buchwald, S. L. J. Organomet. Chem. 1999, 576, 125. (b) Hartwig, J. F. In Modern Amination Methods; Ricci, A., Ed.; Wiley-VCH: Weinheim, Germany, 2000. (c) Muci, A. R.; Buchwald, S. L. Top. Curr. Chem. 2002, 219, 131. (d) Zim, D.; Buchwald, S. L. Org. Lett. 2003, 5, 2413. (e) Urgaonkar, S.; Verkade, J. G. J. Org. Chem. 2004, 69, 9135. (f) Maiti, D.; Buchwald, S. L. J. Am. Chem. Soc. 2009, 131, 17423. (g) Shen, Q.; Hartwig, J. F. Org. Lett. 2008, 10, 4109.

(8) Poola, B.; Choung, W.; Nantz, M. H. Tetrahedron 2008, 64, 10798.

(9) Londregan, A. T.; Jennings, S.; Wei, L. Org. Lett. 2010, 12, 5254.

(10) (a) Dissanayake, A. A.; Staples, R. J.; Odom, A. L. Adv. Synth. Catal. 2014, 356, 1811. (b) Ghorbani-Vaghei, R.; Toghraei-Semiromi, Z.; Karimi-Namib, R. C. R. Chim. 2013, 16, 1111. (c) Gouda, M. A.; Berghot, M. A.; Abd El Ghani, G. E.; Khalil, A. M. Synth. Commun. 2014, 44, 297.

(11) (a) Ibrahim, M. A. El-Sayed Ali T.; El-Gohary, N. M.; El-Kazak, A. M. Eur. J. Chem. 2013, 4, 311. (b) Abdel-Rahman, A. H.; Hammouda, M. A. A.; El-Desoky, S. I. Heteroat. Chem. 2005, 16, 20. (c) Plaskon, A. S.; Grygorenko, O. O.; Ryabukhlin, S. V. Tetrahedron 2012, 68, 2743. (d) Balalaie, S.; Derakhshan-Panaha, F.; Zolfigol, M. A.; Rominger, F. Synlett 2018, 29, 89. 
(12) (a) Chai, H.; Li, J.; Yang, L.; Lu, H.; Zhang, Q.; Shi, D. RSC Adv. 2014, 4, 44811. (b) Wu, J.; Du, X.; Ma, J.; Zhang, Y.; Shi, Q.; Luo, L.; Song, B.; Yangab, S.; Huab, D. Green Chem. 2014, 16, 3210. (c) Liu, X.; Fu, H.; Jiang, Y.; Zhao, Y. Angew. Chem. Int. Ed. 2009, $48,348$.

(13) Kalaria, P. N.; Satasia, S. P.; Avalani, J. R.; Raval, D. K. Eur. J. Med. Chem. 2014, 83, 655.

(14) (a) Oschatz, S.; Brunzel, T.; Wu, X. F.; Langer, P. Org. Biomol. Chem. 2015, 13, 1150. (b) Wu, X.-F.; Oschatz, S.; Block, A.; Spannenberg, A.; Langer, P. Org. Biomol. Chem. 2014, 12, 1865.

(15) (a) Witt, A.; Bergman, J. Curr. Org. Chem. 2003, 7, 659. (b) Mhaske, S. B.; Argade, N. P. Tetrahedron 2006, 62, 9787. (c) Connolly, D. J.; Cusack, D.; O'Sullivan, T. P.; Guiry, P. J. Tetrahedron 2005, 61, 10153.

(16) (a) He, L.; Li, H.; Chen, J.; Wu, X. F. RSC Adv. 2014, 4, 12065. (b) Khan, I.; Ibrar, A.; Abbas, N.; Saeed, A. Eur. J. Med. Chem. 2014, 76, 193.

(17) (a) Tajbakhsh, M.; Ramezanpour, S.; Balalaie, S.; Bijanzadeh, H. R. J. Heterocycl. Chem. 2015, 52, 1559. (b) Fathi Vavsari, V.; Mohammadi Ziarani, G.; Balalaie, S. J. Iran. Chem. Soc. 2016, 13, 1037.

(18) (a) Balalaie, S.; Bararjanian, S.; Hekmat, S.; Salehi, P. Synth. Commun. 2006, 24, 3703. (b) Balalaie, S.; Bararjanian, M.; Hekmat, S.; Salehi, P. Synth. Commun. 2006, 36, 2549. (c) Abdolmohammadi, S.; Balalaie, S. Tetrahedron Lett. 2007, 48, 3299. (d) Balalaie, S.; Abdolmohammadi, S.; Bijanzadeh, H. R.; Amani, A. M. Mol. Diversity 2008, 12, 85. (e) Darvich, F.; Balalaie, S.; Chadegani, F.; Salehi, P. Synth. Commun. 2007, 37, 1059. (f) Balalaie, S.; Bararjanian, M.; Hekmat, S.; Sheikh-Ahmadi, M.; Salehi, P. Synth. Commun. 2007, 37, 1097.

(19) General Procedure for the Synthesis of Functionalized 2Aminopyridines 4a-c:

To a solution of 3-formylchromone ( $1 \mathrm{mmol}, 174 \mathrm{mg}$ ) and malononitrile $(1 \mathrm{mmol}, 66 \mathrm{mg})$ in water $(10 \mathrm{~mL})$ was added diammonium hydrogen phosphate ( $926 \mathrm{mg}, 20 \%$ equiv) and the reaction mixture was stirred at room temperature for $30 \mathrm{~min}$. After formation of the desired chromonyl malononitrile (monitoring by TLC, eluent: $n$-hexane/EtOAc, 3:1). The reaction mixture was stirred at $50{ }^{\circ} \mathrm{C}$ for $3 \mathrm{~h}$. The precipitate was filtered and was washed with water and ethanol.

2-Amino-5-(2-hydroxybenzoyl)nicotinonitrile (4a)

Yield: $222 \mathrm{mg}(93 \%)$; yellow powder; m.p. $198-200{ }^{\circ} \mathrm{C} .{ }^{1} \mathrm{H}$ NMR $\left(300 \mathrm{MHz}\right.$, DMSO- $\left.d_{6}\right): \delta=6.90-6.97(\mathrm{~m}, 2 \mathrm{H}, \mathrm{H}-\mathrm{Ar}), 7.33$ (d, $J=$ $7.5 \mathrm{~Hz}, 1 \mathrm{H}, \mathrm{H}-\mathrm{Ar}$ ), 7.38-7.43 (m, $1 \mathrm{H}, \mathrm{H}-\mathrm{Ar}$ ), 7.81 (br. s, $2 \mathrm{H}, \mathrm{N}-$ $\mathrm{H}), 8.11$ (d, $J=2.4 \mathrm{~Hz}, 1 \mathrm{H}, \mathrm{H}-\mathrm{Py}), 8.47$ (d, $J=2.4 \mathrm{~Hz}, 1 \mathrm{H}, \mathrm{H}-\mathrm{Py})$, 10.25 (br. s, $1 \mathrm{H},-\mathrm{OH}) .{ }^{13} \mathrm{C}$ NMR $\left(75 \mathrm{MHz}\right.$, DMSO- $d_{6}$ ): $\delta=88.9$, $116.1,116.6,119.3,121.8,124.7,130.1,133.0,143.9,155.6$, 156.0, 161.3, 192.1. HRMS (EI): $\mathrm{m} / z$ calcd. for $\mathrm{C}_{13} \mathrm{H}_{9} \mathrm{~N}_{3} \mathrm{O}_{2}[\mathrm{M}]^{+}$ 239.0675; found: 239.0675 .

Colorless crystal (lamina); dimensions $0.150 \times 0.120 \times 0.010$ $\mathrm{mm}^{3}$; crystal system triclinic; space group P1; Z=2; $a=3.7932(9)$ $\AA, b=8.0333(19) \AA, c=19.135(5) \AA, \alpha=79.772(6)^{\circ}, \beta=89.384(6)^{\circ}$, $\gamma=79.103(6)^{\circ} ; V=563.3(2) \AA^{3} ; \rho=1.410 \mathrm{~g} / \mathrm{cm}^{3} ; T=200(2) \mathrm{K}$; $\theta_{\max }=27.514^{\circ}$; radiation Mo $K \alpha, \lambda=0.71073 \AA \AA$; $0.5^{\circ} \omega$-scans with a CCD area detector, covering the asymmetric unit in reciprocal space with a mean redundancy of 2.20 and a completeness of $85.6 \%$ to a resolution of $0.77 \AA, 4556$ reflections measured, 2073 unique $(\mathrm{R}(\mathrm{int})=0.0453), 1868$ observed $(\mathrm{I}>2 \sigma(\mathrm{I}))$. Intensities were corrected for Lorentz and polarization effects, an empirical absorption correction was applied using SADABS based on the Laue symmetry of the reciprocal space, $\mu=0.10 \mathrm{~mm}^{-1}, T_{\min }=0.55, T_{\max }=0.96$. The structure was refined against $\mathrm{F}^{2}$ with a full-matrix least-squares algorithm using the SHELXL-2014/7 (Sheldrick, 2014) software, 168 parameters were refined, hydrogen atoms were treated using appropriate riding models, except $\mathrm{H} 12$ of the hydroxy group, which was refined isotropically. Goodness of fit 1.12 for observed reflections, final residual values $\mathrm{R} 1(\mathrm{~F})=0.049, \quad \mathrm{wR}\left(\mathrm{F}^{2}\right)=0.130$ for observed reflections, residual electron density -0.27 to 0.24 e $\AA^{-3} .{ }^{21}$ CCDC 1496174 contains the supplementary crystallographic data for this paper. These data can be obtained free of charge from The Cambridge Crystallographic Data Centre via www.ccdc.cam.ac.uk/data_request/cif ${ }^{21}$

(20) General Procedure for the Synthesis of Functionalized 2,3Dihydropyrido[2,3-d]pyrimidin-4(1H)-one 6a-o:

A mixture of functionalized 2-amino-pyridine $4 \mathbf{a}-\mathbf{c}(1 \mathrm{mmol})$, aromatic aldehyde $\mathbf{5 a - 1}$ (1.2 $\mathrm{mmol})$, and potassium phosphate $(1.5 \mathrm{mmol}, 340 \mathrm{mg})$ in water $(10 \mathrm{~mL})$ was heated for $7 \mathrm{~h}$ at $100{ }^{\circ} \mathrm{C}$. The precipitate was washed with water and ethanol. 2-(4-Chlorophenyl)-6-(2-hydroxybenzoyl)-2,3-dihydropyrido[2,3-d]pyrimidin-4(1H)-one (6a):

Yellow solid; m.p. $290-293{ }^{\circ} \mathrm{C} \cdot \mathrm{IR}: 3183,3075,1681,1600 \mathrm{~cm}^{-1}$; ${ }^{1} \mathrm{H}$ NMR $\left(300 \mathrm{MHz}\right.$, DMSO- $\left.d_{6}\right): \delta=6.00(\mathrm{~s}, \mathrm{CH}, 1 \mathrm{H}), 6.95(\mathrm{t}, J=$ $7.2 \mathrm{~Hz}, 2 \mathrm{H}, \mathrm{Ar}-\mathrm{H}), 7.30-7.45$ (m, $6 \mathrm{H}, \mathrm{Ar}-\mathrm{H}), 8.14$ (s, $1 \mathrm{H}, \mathrm{Py}-\mathrm{H})$, 8.53 (s, $1 \mathrm{H}, \mathrm{Py}-\mathrm{H}$ ), 8.80 (s, $1 \mathrm{H}, \mathrm{NH}$ ), 8.99 (br. s, $1 \mathrm{H}, \mathrm{NH}$ ), 10.23 (br. s, $1 \mathrm{H}, \mathrm{OH}) .{ }^{13} \mathrm{C}$ NMR $\left(75 \mathrm{MHz}\right.$, DMSO-d $d_{6}$ ): $\delta=64.6,107.6$, 116.5, 119.1, 123.6, 125.4, 128.2, 128.6, 129.7, 132.6, 133.2, 137.1, 140.7, 155.4, 155.9, 158.9, 161.7, 193.4. HRMS (ESI): $\mathrm{m} / \mathrm{z}$ [M-H] $]^{-}$calcd. for $\mathrm{C}_{20} \mathrm{H}_{13}{ }^{35} \mathrm{ClN}_{3} \mathrm{O}_{3}$ : 378.066563; found: 378.06552.

6-(2-Hydroxybenzoyl)-2-(4-nitrophenyl)-2,3-dihydropyrido[2,3-d]pyrimidin-4(1H)-one (6d):

Yellow solid; m.p. $265-268{ }^{\circ} \mathrm{C}$. IR: $3419,3183,1688 \mathrm{~cm}^{-1} \cdot{ }^{1} \mathrm{H}$ NMR (300 MHz, DMSO- $\left.d_{6}\right): \delta=6.14(\mathrm{~s}, \mathrm{CH}, 1 \mathrm{H}), 6.94-6.95(\mathrm{t}, J=$ $7.2 \mathrm{~Hz}, 1 \mathrm{H}, \mathrm{Ar}-\mathrm{H}), 7.29-7.39(\mathrm{~m}, 1 \mathrm{H}, \mathrm{Ar}-\mathrm{H}), 7.69(\mathrm{~d}, J=7.0 \mathrm{~Hz}$, $1 \mathrm{H}$, Ar-H), 8.08-8.27 (m, $4 \mathrm{H}, \mathrm{Ar}-\mathrm{H}), 8.45-8.46$ (d, $J=7.0 \mathrm{~Hz}$, $1 \mathrm{H}, \mathrm{Ar}-\mathrm{H}), 8.54$ (s, $1 \mathrm{H}, \mathrm{Py}-\mathrm{H}), 8.96$ (s, $1 \mathrm{H}, \mathrm{Py}-\mathrm{H}), 9.13$ (s, $1 \mathrm{H}$, $\mathrm{NH}), 10.05(\mathrm{~s}, 1 \mathrm{H}, \mathrm{NH}), 10.14(\mathrm{~s}, 1 \mathrm{H}, \mathrm{OH}) .{ }^{13} \mathrm{C}$ NMR $(75 \mathrm{MHz}$, DMSO- $\left.d_{6}\right): \delta=64.4,116.5,119.3,123.2,124.0,125.4,127.6$, $130.2,130.5,136.1,138.5,147.5,148.8,150.8,155.7,161.7$, 192.3. HRMS (ESI): $m / z \quad[M-H]^{-}$calcd. for $\mathrm{C}_{20} \mathrm{H}_{13} \mathrm{~N}_{4} \mathrm{O}_{5}$ : 389.08906; found: 389.08908

(21) (a) Program SADABS 2012/1 for absorption correction; Sheldrick, G. M.; Bruker Analytical X-ray-Division, Madison, Wisconsin, 2012. (b) Program SHELXL-2014/7; Sheldrick, G. M, 2014; for structure refinement; Acta. Cryst. 2015, C71, 3-8. 